\title{
ON THE EVOLUTION OF COMPACTLY SUPPORTED PLANAR VORTICITY
}

\author{
Dragoş Iftimie \\ IRMAR \\ Université de Rennes 1 \\ Campus de Beaulieu \\ 35042 Rennes \\ FRANCE
}

\author{
Thomas C. Sideris \\ Department of Mathematics \\ University of California \\ Santa Barbara, CA 93106 \\ USA
}

\author{
Pascal Gamblin \\ Département de Mathématiques \\ Université de Paris-Sud \\ Bâtiment 425 \\ 91405 Orsay Cedex \\ FRANCE
}

\section{INTRODUCTION}

The evolution of ideal incompressible fluid vorticity preserves compactness of support. For planar fluids, the diameter of the support of nonnegative initial vorticity will be shown to grow no faster than $\mathcal{O}\left[(t \log t)^{1 / 4}\right]$, improving the bound of $\mathcal{O}\left(t^{1 / 3}\right)$ obtained by Marchioro [I2]. In addition, an example of an initial vorticity with indefinite sign will be given whose support grows unboundedly at a rate of $\mathcal{O}(t)$.

The initial value problem for the $2 \mathrm{~d}$ incompressible Euler equations is globally well-posed in variety of settings. Wolibner [14] established the existence of classical solutions given initial vorticity in $C^{\alpha}\left(\mathbb{R}^{2}\right) \cap L^{1}\left(\mathbb{R}^{2}\right)$, and Yudovitch [15] gave the framework for weak solutions starting with initial vorticity in $L^{1}\left(\mathbb{R}^{2}\right) \cap L^{\infty}\left(\mathbb{R}^{2}\right)$. Three basic elements of the classical existence theory are relevant for the present discussion. The divergence-free fluid velocity vector 
field $v(t, x)$ generates a particle flow map $\Phi(t, p)$ through the system of ODE's

$$
\frac{d}{d t} \Phi(t, p)=v(t, \Phi(t, p)), \quad \Phi(0, p)=p
$$

such that the map $p \mapsto \Phi(t, p)$ is a continuously varying family of areapreserving diffeomorphisms of the plane. The scalar vorticity $\omega=\partial_{1} v_{2}-\partial_{2} v_{1}$ is transported by this flow

$$
D_{t} \omega=\partial_{t} \omega+v \cdot \nabla \omega=0, \quad \omega(0, x)=\omega_{0}(x),
$$

and the velocity is coupled to the vorticity through the Biot-Savart law

$$
v(t, x)=\frac{1}{2 \pi} \int_{\mathbb{R}^{2}} \frac{(x-y)^{\perp}}{|x-y|^{2}} \omega(t, y) d y .
$$

Existence, but not uniqueness, of global weak solutions has been established for less regular initial vorticity of compact support. DiPerna and Majda [9] treated the case of initial data in $L^{p}\left(\mathbb{R}^{2}\right)$, for $1<p<\infty$, and Delort [8] tackled vortex sheet initial vorticity, a positive Radon measure in $H^{-1}\left(\mathbb{R}^{2}\right)$.

Despite this successful existence theory, little can be said about the large time behavior of solutions. This is not surprising since point vortex approximations, even using small numbers of particles, can generate complex dynamics. Given that the vorticity is transported by a area-preserving flow (2), it follows that its $L^{p}$ norms are constant in time. In the case of smooth data, Hölder regularity of the flow map is preserved in time, but the Hölder norm of the flow map is only known to be bounded by an expression of the form $\exp (\exp C t)$. Clearly, any growth in the Hölder norm of the flow map would be related to the evolution of compact regions under the flow.

If the initial vorticity is supported in a compact set $\Omega \subset \mathbb{R}^{2}$, then equation (2) shows that at time $t>0$ the vorticity is supported in $\Omega(t)=\Phi(t, \Omega)$. Nothing can be said about the geometry of $\Omega(t)$. However in the case where the vorticity equals the characteristic function of a set with smooth boundary, the so-called vortex patch, Chemin [5] proved that the regularity of the boundary is propagated, see also [3], []. A simple estimate from (3), given in Lemma 2.1 below, provides a uniform bound for the velocity, and so the support of the vorticity can grow at most linearly in time. For positive initial vorticity, Marchioro [1:2] demonstrated that the conservation of the moment of inertia, $\int_{\mathbb{R}^{2}}|x|^{2} \omega(t, x) d x$, further acts to constrain the spreading of the support 
to a rate of $\mathcal{O}\left(t^{1 / 3}\right)$. This result was generalized to include vorticity in $L^{p}$ for $2<p<\infty$, in [I]].

Here it will be shown, in Theorem 2.1, that Marchioro's bound for the growth rate of the support of positive vorticity can be improved to $\mathcal{O}\left[(t \log t)^{1 / 4}\right]$ by taking into account not only the conservation of the moment of inertia but also the conservation of the center of mass, $\int_{\mathbb{R}^{2}} x \omega(t, x) d x$. As in [12], bounds for the flow map will come from an estimate for the radial component of the velocity starting from (3). The heart of the matter is to measure the vorticity in $L^{1}$ outside of balls centered at the origin, Proposition 2.1. The approach taken here, which is the second main difference with [12], is to derive a differential inequality for a certain smooth approximation to this $L^{1}$ quantity. The analysis applies to weak solutions in $L^{p}, 2<p \leq \infty$. Another proof of Proposition 2.1, using higher momenta, has been found by Gamblin. His argument is included in the appendix at the end of the article. After the completion of this work, we learned that Serfati [13] has independently obtained a result similar to Theorem 2.1 with the factor $t^{1 / 4} \log \circ \cdots \circ \log t$ replacing $(t \log t)^{1 / 4}$.

There are a few examples of explicit solutions, but none of these exhibit any growth of support. Spherically symmetric initial vorticity gives rise to a stationary solution whose velocity vector field induces flow lines which follow circles about the origin. The support of the Kirchoff elliptical vortex patch rotates with constant angular velocity, although the velocity vector field has a nontrivial structure exterior to the support, (see [10], p.232). The support of the so-called Batchelor couple, the continuous analog of a pair of oppositely charged point vortices, moves by translation with speed $\mathcal{O}(t)$, without any change of shape, (see [2], p.534). On the other hand, numerical simulations starting with a pair of positively charged vortex patches show homogenization of the patches simultaneous with the formation of long filaments [4].

In the final section, an example is presented for which the support of the vorticity grows at a rate of $\mathcal{O}(t)$. This rate is optimal since, as mentioned above, the growth can be at most linear in time. The initial vorticity is not positive, rather it consists of four blobs, identical except for alternating sign, located symmetrically in the four quadrants. The initial configuration is inspired by two examples. First, the discrete analog of this set-up can be integrated explicitly, and the point vortices are seen to spread at a rate of 
$\mathcal{O}(t)$. Secondly, at the other extreme, Bahouri and Chemin [I] consider an example for which the initial vorticity is piecewise constant with alternating values \pm 1 in the unit square of the four quadrants. There one finds rapid loss of Hölder regularity of the flow map. The motion in our example restricts to a solution of the Euler equations in the first quadrant with slip boundary conditions. The proof will show that the center of the mass located in the first quadrant moves at a rate of $\mathcal{O}(t)$. In this case, the conservation of the center of mass and moment of inertia are no longer useful since both quantities vanish. Instead, we shall use conservation of energy.

\section{When The VORTICITY IS POSITIVE}

In this section we prove that the support of nonnegative vorticity in $L^{\infty}$ grows no faster than $C(t \log t)^{1 / 4}$. We will make use of several quantities that are conserved by the time evolution, namely the total mass

$$
\int \omega(t, x) d x=\int \omega_{0}(x) d x=m_{0},
$$

the maximum norm

$$
\|\omega(t)\|_{L^{\infty}}=\left\|\omega_{0}\right\|_{L^{\infty}}=M_{0},
$$

the center of mass

$$
m_{0}^{-1} \int x \omega(t, x) d x=m_{0}^{-1} \int x \omega_{0}(x) d x=c_{0},
$$

and the moment of inertia

$$
\int|x|^{2} \omega(t, x) d x=\int|x|^{2} \omega_{0}(x) d x=i_{0} .
$$

Assume that the support of $\omega_{0}$ is contained in the ball centered at the origin of radius $d_{0}$. We are going to prove the following theorem.

Theorem 2.1. Let $\omega(t, x)$ be the solution of the 2d incompressible Euler equations with a positive compactly supported initial vorticity $\omega_{0} \in L^{\infty}\left(\mathbb{R}^{2}\right)$. There exists a constant $C_{0}=C_{0}\left(i_{0}, d_{0}, m_{0}, M_{0}\right)$ such that, for every time $t \geq 0$, the support of $\omega(t, \cdot)$ is contained in the ball $|x|<4 d_{0}+C_{0}[t \log (2+t)]^{1 / 4}$.

Proof. First, by making the change of variable $x \rightarrow x-c_{0}$, we may assume, without loss of generality, that the center of mass is located at the origin.

In the following estimates, constants will be independent of $\omega_{0}$, unless otherwise indicated, and then the dependence will be only through the quantities $i_{0}$, 
$d_{0}, m_{0}$, and $M_{0}$. We will establish the theorem for classical solutions, and the general result, for weak solutions, follows immediately since these quantities are stable under passage to the weak limit. The time variable will often be suppressed since it plays no role in the estimation of the various convolution integrals.

We are going to show that the radial component of the velocity satisfies an estimate of the form

$$
\left|\frac{x}{|x|} \cdot v(t, x)\right| \leq \frac{C_{0}}{|x|^{3}}, \quad \text { for all } \quad|x| \geq 4 d_{0}+C_{0}[t \log (2+t)]^{1 / 4}
$$

with $C_{0}=C_{0}\left(i_{0}, d_{0}, m_{0}, M_{0}\right)$. The proof of the theorem concludes by noticing that the region

$$
\left\{(t, x): t \geq 0,|x|<4 d_{0}+C_{0}[t \log (2+t)]^{1 / 4}\right\}
$$

is invariant for the flow

$$
\frac{d t}{d s}=1, \quad \frac{d x}{d s}=v(t, x)
$$

since the bound (4) implies that the vector field $(1, v(t, x))$ points inward along the boundary of this region.

We now turn to the verification of (4). The radial part of the velocity is

$$
\frac{x}{|x|} \cdot v(x)=\frac{1}{2 \pi} \int \frac{x}{|x|} \cdot \frac{(x-y)^{\perp}}{|x-y|^{2}} \omega(y) d y,
$$

in which $\left(x_{1}, x_{2}\right)^{\perp}=\left(-x_{2}, x_{1}\right)$. The last integral will be divided into two pieces.

The portion of the integral over the region $|x-y|<|x| / 2$ is immediately seen to be bounded by

$$
C \int_{|x-y|<|x| / 2} \frac{\omega(y)}{|x-y|} d y
$$

Using that $x \cdot(x-y)^{\perp}=-x \cdot y^{\perp}$ and the fact that the center of mass is at the origin, we can express the other portion as 


$$
\begin{gathered}
\int_{|x-y|>|x| / 2} \frac{x}{|x|} \cdot \frac{(x-y)^{\perp}}{|x-y|^{2}} \omega(y) d y=-\int_{|x-y|>|x| / 2} \frac{x \cdot y^{\perp}}{|x||x-y|^{2}} \omega(y) d y \\
=-\int_{|x-y|>|x| / 2} \frac{x \cdot y^{\perp}}{|x|}\left(\frac{1}{|x-y|^{2}}-\frac{1}{|x|^{2}}\right) \omega(y) d y \\
+\int_{|x-y|<|x| / 2} \frac{x \cdot y^{\perp}}{|x|^{3}} \omega(y) d y \\
=-\int_{|x-y|>|x| / 2} \frac{x \cdot y^{\perp}}{|x|} \frac{\langle y, 2 x-y\rangle}{|x-y|^{2}|x|^{2}} \omega(y) d y \\
+\int_{|x-y|<|x| / 2} \frac{x \cdot y^{\perp}}{|x|^{3}} \omega(y) d y .
\end{gathered}
$$

Next, we note that $|x-y|>|x| / 2$ implies

$$
|2 x-y| \leq|x-y|+|x|<3|x-y|,
$$

and so the first of these integrals is bounded as follows

$$
\begin{aligned}
\int_{|x-y|>|x| / 2} \frac{x \cdot y^{\perp}}{|x|} \frac{\langle y, 2 x-y\rangle}{|x-y|^{2}|x|^{2}} \omega(y) d y \mid & \leq \int_{|x-y|>|x| / 2} \frac{|y|^{2}|2 x-y|}{|x|^{2}|x-y|^{2}} \omega(y) d y \\
& \leq \frac{C}{|x|^{3}} \int_{|x-y|>|x| / 2}|y|^{2} \omega(y) d y \leq \frac{C i_{0}}{|x|^{3}} .
\end{aligned}
$$

On the grounds of simple homogeneity, it is difficult to see how to improve this estimate using only the conserved quantities at hand.

As for the second piece, we use that $|x-y|<|x| / 2$ gives $|y| \leq 3|x| / 2$ to write

$$
\left|\int_{|x-y|<|x| / 2} \frac{x \cdot y^{\perp}}{|x|^{3}} \omega(y) d y\right| \leq C \int_{|x-y|<|x| / 2} \frac{\omega(y)}{|x-y|} d y .
$$

We have deduced the following estimate for the radial component of velocity

$$
\left|\frac{x}{|x|} \cdot v(x)\right| \leq \frac{C i_{0}}{|x|^{3}}+C \int_{|x-y|<|x| / 2} \frac{\omega(y)}{|x-y|} d y .
$$

The rest of the proof consists in showing that the last integral is negligible for large $|x|$. 
Before going any further, we need an elementary lemma which gives a bound of the $L^{\infty}$ norm of the velocity in terms of the vorticity.

Lemma 2.1. Let $S \subset \mathbb{R}^{2}$ and $h: S \rightarrow \mathbb{R}^{+}$be a function belonging to $L^{1}(S) \cap$ $L^{\infty}(S)$. Then

$$
\int_{S} \frac{h(y)}{|x-y|} d y \leq C\|h\|_{L^{1}(S)}^{1 / 2}\|h\|_{L^{\infty}(S)}^{1 / 2}
$$

Proof of Lemma 2.1. Let $k$ be an arbitrary positive number. We can write

$$
\begin{aligned}
\int_{S} \frac{h(y)}{|x-y|} d y & =\int_{S \cap\{|x-y|>k\}} \frac{h(y)}{|x-y|} d y+\int_{S \cap\{|x-y|<k\}} \frac{h(y)}{|x-y|} d y \\
& \leq \frac{\|h\|_{L^{1}(S)}}{k}+\|h\|_{L^{\infty}(S)} \int_{|x-y|<k} \frac{1}{|x-y|} d y \\
& =\frac{\|h\|_{L^{1}(S)}}{k}+\|h\|_{L^{\infty}(S)} 2 \pi k .
\end{aligned}
$$

Choose $k=\|h\|_{L^{1}(S)}^{1 / 2}\|h\|_{L^{\infty}(S)}^{-1 / 2}$.

Therefore, from (5), Lemma 2.1, and the fact that

$$
\{y:|x-y|<|x| / 2\} \subset\{y:|y|>|x| / 2\},
$$

the estimate for the radial component of the velocity is

$$
\left|\frac{x}{|x|} \cdot v(x)\right| \leq \frac{C i_{0}}{|x|^{3}}+C M_{0}^{1 / 2}\left(\int_{|y|>|x| / 2} \omega(y) d y\right)^{1 / 2} .
$$

Given the following proposition, with $k=6$, the last integral is also $\mathcal{O}\left(|x|^{-3}\right)$ for $|x|$ large so that inequality (4) holds, and hence Theorem 2.1 is valid.

Proposition 2.1. There exists a constant $C_{0}=C_{0}\left(i_{0}, d_{0}, m_{0}, M_{0}, k\right)$ such that for any $k>0$

$$
\int_{|y|>|x| / 2} \omega(t, y) d y \leq \frac{C_{0}}{|x|^{k}}
$$

for all $|x|>4 d_{0}+C_{0}[t \log (2+t)]^{1 / 4}$.

Proof of the Proposition. Let us consider the function

$$
f_{r}(t)=\int \phi_{r}(y) \omega(t, y) d y
$$

in which

$$
\phi_{r}(y)=\eta\left(\frac{|y|^{2}-r^{2}}{\lambda r^{2}}\right)
$$


with

$$
\eta(s)=\frac{e^{s}}{1+e^{s}},
$$

and $\lambda=\lambda(r)$ is a positive function less than 1 to be chosen in (18). We shall use later that

$$
\eta^{\prime}(s)=e^{s} /\left(1+e^{s}\right)^{2} \leq\left\{\begin{array}{l}
\eta(s) \\
e^{-|s|}
\end{array}\right.
$$

and

$$
\eta^{\prime \prime}(s)=e^{s}\left(1-e^{s}\right) /\left(1+e^{s}\right)^{3}, \quad \text { so } \quad\left|\eta^{\prime \prime}\right| \leq \eta
$$

If $|y|>r$, then $\left(|y|^{2}-r^{2}\right) / r^{2} \lambda>0$, and the monotonicity of $\eta$ implies that

$$
f_{r}(t)>\eta(0) \int_{|y|>r} \omega(t, y) d y .
$$

Hence, in order to prove the Proposition, it suffices to show that there is a constant $C_{0}=C_{0}\left(i_{0}, d_{0}, m_{0}, M_{0}, k\right)$ such that

$$
f_{r}(t) \leq \frac{C_{0}}{r^{k}}
$$

as long as $r>2 d_{0}+C_{0}[t \log (2+t)]^{1 / 4}$. The inequality of the Proposition follows by setting $r=|x| / 2$. The rest of the section is concerned with the proof of the inequality (6). (For notational convenience, the integration variable of $f_{r}(t)$ will now be switched from $y$ to $x$.)

Differentiating $f_{r}(t)$, using the vorticity equation (2), integrating by parts, and finally using the Biot-Savart law (3) gives

$$
\begin{aligned}
f_{r}^{\prime}(t) & =\int \phi_{r}(x) \partial_{t} \omega(t, x) d x \\
& =-\int \phi_{r}(x) v(t, x) \cdot \nabla \omega(t, x) d x \\
& =\int \nabla \phi_{r}(x) \cdot v(t, x) \omega(t, x) d x \\
& =\frac{1}{2 \pi} \iint \nabla \phi_{r}(x) \cdot \frac{(x-y)^{\perp}}{|x-y|^{2}} \omega(t, x) \omega(t, y) d x d y \\
& =\frac{1}{2 \pi} \iint 2 \eta^{\prime}\left(\frac{|x|^{2}-r^{2}}{\lambda r^{2}}\right) \frac{\left\langle x,(x-y)^{\perp}\right\rangle}{\lambda r^{2}|x-y|^{2}} \omega(t, x) \omega(t, y) d x d y .
\end{aligned}
$$


Furthermore, since the center of mass is at the origin, we have

$$
\begin{aligned}
f_{r}^{\prime}(t)=\frac{1}{\pi} \iint \eta^{\prime}\left(\frac{|x|^{2}-r^{2}}{\lambda r^{2}}\right) & \frac{\left\langle x,(x-y)^{\perp}\right\rangle}{\lambda r^{2}} \\
& \times\left(\frac{1}{|x-y|^{2}}-\frac{1}{|x|^{2}}\right) \omega(t, x) \omega(t, y) d x d y .
\end{aligned}
$$

Let us denote by $L(x, y)$ the kernel in the integral above.

Define the following sets

$$
\begin{aligned}
& \Omega_{1}=\left\{(x, y):|x|<\frac{r}{2} \text { or }|x|>\frac{3 r}{2}\right\}, \\
& \Omega_{2}=\left\{(x, y): \frac{r}{2} \leq|x| \leq \frac{3 r}{2},|y|<\frac{r}{4} \text { or }|y|>\frac{7 r}{4}\right\}, \\
& \Omega_{3}=\left\{(x, y): \frac{r}{2} \leq|x| \leq \frac{3 r}{2}, \frac{r}{4} \leq|y| \leq \frac{7 r}{4}\right\}, \\
& \Omega_{4}=\left\{(x, y): \frac{r}{4}<|x|<\frac{7 r}{4}, \frac{r}{4}<|y|<\frac{7 r}{4}\right\} .
\end{aligned}
$$

Clearly, the whole space $\mathbb{R}^{2} \times \mathbb{R}^{2}$ can be written as the disjoint union $\Omega_{1} \cup$ $\Omega_{2} \cup \Omega_{3}$. Therefore, we have

$$
f_{r}^{\prime}=\iint_{\Omega_{1}} L(x, y) d x d y+\iint_{\Omega_{2}} L(x, y) d x d y+\iint_{\Omega_{3}} L(x, y) d x d y .
$$

Moreover, since $\Omega_{3} \subset \Omega_{4}$, we can further write

$$
\begin{aligned}
f_{r}^{\prime}=\iint_{\Omega_{1}} L(x, y) d x d y+\iint_{\Omega_{2}} L(x, y) d x d y & \\
& \quad+\iint_{\Omega_{4}} L(x, y) d x d y-\iint_{\Omega_{4} \backslash \Omega_{3}} L(x, y) d x d y .
\end{aligned}
$$

Now, $\Omega_{4} \backslash \Omega_{3} \subset \Omega_{1}$ where $|x|<r / 2$ or $|x|>3 r / 2$ hold. In this case, since $\eta^{\prime}(s)$ is bounded by $e^{-|s|}$, we have $\eta^{\prime}\left(\frac{|x|^{2}-r^{2}}{\lambda r^{2}}\right) \leq e^{-1 / 2 \lambda}$. Therefore, in the region $\Omega_{1}$ we have the bound

$$
\begin{aligned}
|L(x, y)| & =\frac{1}{\pi \lambda r^{2}}\left|\eta^{\prime}\left(\frac{|x|^{2}-r^{2}}{\lambda r^{2}}\right)\right| \\
& \times\left|\frac{\left\langle x,(x-y)^{\perp}\right\rangle}{|x-y|^{2}}+\frac{\left\langle x, y^{\perp}\right\rangle}{|x|^{2}}\right| \omega(x) \omega(y) \\
\leq & \frac{C}{\lambda r^{2} e^{1 / 2 \lambda}}\left(\frac{|x|}{|x-y|}+\frac{|y|}{|x|}\right) \omega(x) \omega(y) .
\end{aligned}
$$


An application of the Cauchy-Schwarz inequality yields

$$
\int|x| \omega(x) d x \leq\left(\int \omega(x) d x\right)^{1 / 2}\left(\int|x|^{2} \omega(x) d x\right)^{1 / 2}=\left(m_{0} i_{0}\right)^{1 / 2} .
$$

From (10), applying Lemma 2.1 and inequality (11) gives

$$
\begin{aligned}
& \left|\iint_{\Omega_{1}} L(x, y) d x d y-\iint_{\Omega_{4} \backslash \Omega_{3}} L(x, y) d x d y\right| \\
& \leq \frac{C}{\lambda r^{2} e^{1 / 2 \lambda}}\left[\left(\int \frac{\omega(x)}{|x|} d x\right)\left(\int|y| \omega(y) d y\right)\right. \\
& \left.\quad+\int|x| \omega(x)\left(\int \frac{\omega(y)}{|x-y|} d y\right) d x\right] \\
& \leq \frac{C}{\lambda r^{2} e^{1 / 2 \lambda}} m_{0}\left(M_{0} i_{0}\right)^{1 / 2} .
\end{aligned}
$$

Assume now that $(x, y) \in \Omega_{2}$. This implies that $|x-y|>r / 4$ and also that $|2 x-y| \leq|x|+|x-y| \leq 7|x-y|$. Thus, since $\eta^{\prime} \leq \eta$, it follows that on $\Omega_{2}$

$$
\begin{aligned}
|L(x, y)| & =\frac{1}{\pi} \eta^{\prime}\left(\frac{|x|^{2}-r^{2}}{\lambda r^{2}}\right) \frac{\left|\left\langle x, y^{\perp}\right\rangle\right|}{\lambda r^{2}} \frac{|\langle y, 2 x-y\rangle|}{|x|^{2}|x-y|^{2}} \omega(x) \omega(y) \\
& \leq C \phi_{r}(x) \frac{|y|^{2}}{\lambda r^{2}|x||x-y|} \omega(x) \omega(y) \\
& \leq \frac{C}{\lambda r^{4}} \phi_{r}(x) \omega(x)|y|^{2} \omega(y) .
\end{aligned}
$$

Finally, from the conservation of the moment of inertia, we deduce

$$
\left|\iint_{\Omega_{2}} L(x, y) d x d y\right| \leq \iint \frac{C}{\lambda r^{4}} \phi_{r}(x) \omega(x)|y|^{2} \omega(y) d x d y=\frac{C i_{0}}{\lambda r^{4}} f_{r} .
$$

It remains to study the integral over $\Omega_{4}$. Note that this domain is symmetric with respect to the diagonal. Interchanging the roles of the coordinates, we may write

$$
\iint_{\Omega_{4}} L(x, y) d x d y=\frac{1}{2} \iint_{\Omega_{4}}[L(x, y)+L(y, x)] d x d y .
$$

But it is easy to see that $L(x, y)+L(y, x)=L_{1}(x, y)+L_{2}(x, y)$ where

$$
\begin{aligned}
L_{1}(x, y)=\frac{1}{\pi \lambda r^{2}} & {\left[\eta^{\prime}\left(\frac{|x|^{2}-r^{2}}{\lambda r^{2}}\right)\left\langle x,(x-y)^{\perp}\right\rangle\right.} \\
& \left.-\eta^{\prime}\left(\frac{|y|^{2}-r^{2}}{\lambda r^{2}}\right)\left\langle y,(x-y)^{\perp}\right\rangle\right]\left(\frac{1}{|x-y|^{2}}-\frac{1}{|x|^{2}}\right) \omega(x) \omega(y)
\end{aligned}
$$


and

$$
L_{2}(x, y)=\frac{1}{\pi \lambda r^{2}} \eta^{\prime}\left(\frac{|y|^{2}-r^{2}}{\lambda r^{2}}\right)\left\langle y,(x-y)^{\perp}\right\rangle\left(\frac{1}{|y|^{2}}-\frac{1}{|x|^{2}}\right) \omega(x) \omega(y)
$$

If $(x, y)$ lies in $\Omega_{4}$, then $|x| \sim r$ and $|y| \sim r$. Recalling that $\eta^{\prime} \leq \eta$, we can bound $L_{2}(x, y)$ by

$$
\begin{aligned}
\left|L_{2}(x, y)\right| & \leq \frac{C}{\lambda r^{2}} \phi_{r}(x)|y||x-y|\left(\frac{1}{|x|^{2}}+\frac{1}{|y|^{2}}\right) \omega(x) \omega(y) . \\
& \leq \frac{C}{\lambda r^{2}} \phi_{r}(x) \omega(x) \omega(y)
\end{aligned}
$$

We now go to the estimate of $L_{1}(x, y)$. The observation that

$$
\left\langle x,(x-y)^{\perp}\right\rangle=\left\langle y,(x-y)^{\perp}\right\rangle
$$

allows $L_{1}$ to be rewritten as

$$
\begin{aligned}
L_{1}(x, y)=\frac{1}{\pi \lambda r^{2}}\left[\eta^{\prime}\left(\frac{|x|^{2}-r^{2}}{\lambda r^{2}}\right)-\eta^{\prime}\left(\frac{|y|^{2}-r^{2}}{\lambda r^{2}}\right)\right] \\
\quad \times\left\langle y,(x-y)^{\perp}\right\rangle\left(\frac{1}{|x-y|^{2}}-\frac{1}{|x|^{2}}\right) \omega(x) \omega(y) .
\end{aligned}
$$

The mean value theorem ensures the existence of a point $\xi$ located between $\left(|x|^{2}-r^{2}\right) / \lambda r^{2}$ and $\left(|y|^{2}-r^{2}\right) / \lambda r^{2}$ such that

$$
\eta^{\prime}\left(\frac{|x|^{2}-r^{2}}{\lambda r^{2}}\right)-\eta^{\prime}\left(\frac{|y|^{2}-r^{2}}{\lambda r^{2}}\right)=\eta^{\prime \prime}(\xi) \frac{|x|^{2}-|y|^{2}}{\lambda r^{2}}
$$

Since $\left|\eta^{\prime \prime}\right| \leq \eta$, we infer from the monotonicity of $\eta$ that

$$
\begin{aligned}
\mid \eta^{\prime}\left(\frac{|x|^{2}-r^{2}}{\lambda r^{2}}\right) & -\eta^{\prime}\left(\frac{|y|^{2}-r^{2}}{\lambda r^{2}}\right) \mid \\
& \leq \frac{C}{\lambda r^{2}}\left[\eta\left(\frac{|x|^{2}-r^{2}}{\lambda r^{2}}\right)+\eta\left(\frac{|y|^{2}-r^{2}}{\lambda r^{2}}\right)\right]|x-y||x+y| \\
& =\frac{C}{\lambda r^{2}}\left[\phi_{r}(x)+\phi_{r}(y)\right]|x-y||x+y| .
\end{aligned}
$$


We are now able to estimate $L_{1}(x, y)$. From relation (15) and the preceding inequality we get

$$
\begin{aligned}
\left|L_{1}(x, y)\right| \leq & \frac{C}{\lambda^{2} r^{4}}\left[\phi_{r}(x)+\phi_{r}(y)\right]|x-y||x+y|\left|\left\langle y,(x-y)^{\perp}\right\rangle\right| \\
& \times \frac{|\langle y, 2 x-y\rangle|}{|x|^{2}|x-y|^{2}} \omega(x) \omega(y) \\
\leq & \frac{C}{\lambda^{2} r^{4}}\left[\phi_{r}(x)+\phi_{r}(y)\right]|x-y|^{2}|x+y||y| \\
\quad \times \frac{|y||2 x-y|}{|x|^{2}|x-y|^{2}} \omega(x) \omega(y) & \\
= & \frac{C}{\lambda^{2} r^{4}}\left[\phi_{r}(x)+\phi_{r}(y)\right] \frac{|x+y||y|^{2}|2 x-y|}{|x|^{2}} \omega(x) \omega(y) .
\end{aligned}
$$

Again using that $|x| \sim r$ and $|y| \sim r$ when $(x, y) \in \Omega_{4}$, we get

$$
\left|L_{1}(x, y)\right| \leq \frac{C}{\lambda^{2} r^{2}}\left[\phi_{r}(x)+\phi_{r}(y)\right] \omega(x) \omega(y) .
$$

The final estimate for the integral over $\Omega_{4}$ now comes by using the estimate above and inequality (14). We get

$$
\left|L_{1}(x, y)+L_{2}(x, y)\right| \leq \frac{C}{\lambda^{2} r^{2}}\left[\phi_{r}(x)+\phi_{r}(y)\right] \omega(x) \omega(y)
$$

and hence,

$$
\begin{aligned}
\mid \iint_{\Omega_{4}} L(x, & y) d x d y \mid \\
& \leq \iint_{\Omega_{4}} \frac{C}{\lambda^{2} r^{2}}\left[\phi_{r}(x)+\phi_{r}(y)\right] \omega(x) \omega(y) d x d y \\
& =2 \frac{C}{\lambda^{2} r^{2}} \iint_{\Omega_{4}} \phi_{r}(x) \omega(x) \omega(y) d x d y \\
& \leq \frac{C}{\lambda^{2} r^{2}} \int \phi_{r}(x) \omega(x) d x \int_{\frac{r}{4}<|y|<\frac{7 r}{4}} \omega(y) d y \\
& \leq \frac{C i_{0}}{\lambda^{2} r^{4}} f_{r}
\end{aligned}
$$

where we used the conservation of moment of inertia to deduce that

$$
\int_{r / 4<|y|<7 r / 4} \omega(y) d y \leq \frac{C i_{0}}{r^{2}} .
$$


Putting together relations (9), (12), (13) and (16) we obtain the following differential inequality

$$
f_{r}^{\prime}(t) \leq \frac{C i_{0}}{\lambda^{2} r^{4}} f_{r}(t)+\frac{C}{\lambda r^{2} e^{1 / 2 \lambda}} m_{0}\left(M_{0} i_{0}\right)^{1 / 2} .
$$

Gronwall's lemma implies

$$
f_{r}(t) \leq f_{r}(0) \exp \left[\frac{C i_{0}}{\lambda^{2} r^{4}} t\right]+m_{0} \lambda r^{2}\left(\frac{M_{0}}{i_{0}}\right)^{1 / 2} \exp \left[\frac{C i_{0}}{\lambda^{2} r^{4}} t-\frac{1}{2 \lambda}\right] .
$$

If we assume that $r \geq 2 d_{0}$, then from the definition of $f_{r}(t)$, we have that

$$
f_{r}(0) \leq m_{0} e^{-1 / 2 \lambda}
$$

which gives us the bound

$$
f_{r}(t) \leq m_{0}\left(1+\lambda r^{2}\left(\frac{M_{0}}{i_{0}}\right)^{1 / 2}\right) \exp \left[\frac{C i_{0}}{\lambda^{2} r^{4}} t-\frac{1}{2 \lambda}\right] .
$$

Now we suppose that

$$
t \leq \frac{\lambda r^{4}}{4 C i_{0}}
$$

so that

$$
\frac{C i_{0}}{\lambda^{2} r^{4}} t-\frac{1}{2 \lambda} \leq-\frac{1}{4 \lambda}
$$

and we make the choice

$$
\lambda=\left[4(k+2) \log \left(\frac{r}{d_{0}}\right)\right]^{-1} .
$$

Combining the above, we have shown that

$$
f_{r}(t) \leq \frac{C_{0}}{r^{k}}
$$

with $C_{0}=C_{0}\left(i_{0}, d_{0}, m_{0}, M_{0}, k\right)$, provided that $r \geq 2 d_{0}$, (17), and (18) hold. The conclusion of Proposition 2.1 follows by noting that, for $C_{0}$ large enough, $t$ and $r$ will lie in the appropriate range as long as

$$
r \geq 2 d_{0}+C_{0}[t \log (2+t)]^{1 / 4} .
$$

We remark that the proof goes through for $\omega_{0} \in L^{p}, 2<p \leq \infty$, simply by replacing the maximum norm by the $L^{p}$-norm in Lemma 2.1 and remembering that the $L^{p}$-norm is conserved under the time evolution. 


\section{An eXAMPle of Linear GROWTH}

The aim of this section is to present an example of vorticity, with indefinite sign, whose support grows like $\mathcal{O}(t)$. Let us denote the first quadrant by $\mathbf{Q}$. Let $\widetilde{\omega}_{0}$ be a nonnegative function, belonging to $L^{\infty}$, copactly supported in Q. We denote $m_{0}=\int \widetilde{\omega}_{0}(x) d x, M_{0}=\left\|\widetilde{\omega}_{0}\right\|_{L^{\infty}}$, and $\mathbf{P}_{0}=\int x \widetilde{\omega}_{0}(x) d x$. Our example of initial vorticity is a function antisymmetric with respect with both coordinate axes and equal to $\widetilde{\omega}_{0}$ in the first quadrant. In other words, using $\bar{x}$ for the complex conjugate of $x$, we define $\omega_{0}(x)=\widetilde{\omega}_{0}(x)$ for $x \in \mathbf{Q}$ and extend $\omega_{0}$ to $\mathbb{R}^{2}$ so as to have $\omega_{0}(x)=-\omega_{0}(\bar{x})=-\omega_{0}(-\bar{x})=\omega_{0}(-x)$. We shall prove the following theorem.

Theorem 3.1. There exists a constant $C_{0}=C_{0}\left(m_{0}, M_{0}, \mathbf{P}_{0}\right)$ such that, for every time $t$, the diameter, $d(t)$, of the support of the vorticity evolved from $\omega_{0}$ satisfies $d(t) \geq C_{0} t$.

Proof. By uniqueness, the vorticity $\omega(t, x)$ preserves the antisymmetry of the initial data,

$$
\omega(t, x)=-\omega(t, \bar{x})=-\omega(t,-\bar{x})=\omega(t,-x) .
$$

Moreover, the flow map is antisymmetric, and so it leaves each quadrant and both coordinate axes invariant. Consequently, we have

$$
\int_{\mathbf{Q}} \omega(t, x) d x=\int_{\mathbf{Q}} \omega(0, x) d x=\int_{\mathbf{Q}} \widetilde{\omega}_{0}(x) d x=m_{0} .
$$

We shall consider the evolution of the center of mass of $\omega(t, x)$ restricted to $\mathrm{Q}$ defined by

$$
\mathbf{P}(t)=\frac{1}{m_{0}} \int_{\mathbf{Q}} x \omega(t, x) d x .
$$

Let $\mathbf{P}(t)=\left(P_{1}(t), P_{2}(t)\right)$. The support of $\omega$ has a non-empty intersection with the region $\left\{x_{1} \geq P_{1}\right\}$. Therefore, the symmetry properties of $\omega(t, x)$ imply that the diameter of the support of the vorticity is bounded by below by $P_{1}(t)$. So, in order to prove Theorem 3.1 , it is enough to prove that $P_{1}(t) \geq C_{0}\left(m_{0}, M_{0}, \mathbf{P}_{0}\right) t$. In the course of the proof, we shall also see that $P_{1}(t)$ is increasing and that $P_{2}(t)$ is decreasing. 
From the Biot-Savart law (3) along with the obvious changes of coordinates, we deduce

$$
\begin{aligned}
v(x) & =\int_{\mathbb{R}^{2}} \frac{(x-y)^{\perp}}{|x-y|^{2}} \omega(y) d y \\
& =\int_{\mathbf{Q}}\left(\frac{(x-y)^{\perp}}{|x-y|^{2}}+\frac{(x+y)^{\perp}}{|x+y|^{2}}-\frac{(x-\bar{y})^{\perp}}{|x-\bar{y}|^{2}}-\frac{(x+\bar{y})^{\perp}}{|x+\bar{y}|^{2}}\right) \omega(y) d y .
\end{aligned}
$$

Separating the components, we can further write

$$
\begin{aligned}
v_{1}(x)=\int_{\mathbf{Q}}\left[-\left(x_{2}-y_{2}\right)\left(\frac{1}{|x-y|^{2}}-\frac{1}{|x+\bar{y}|^{2}}\right)\right. \\
\left.+\left(x_{2}+y_{2}\right)\left(\frac{1}{|x-\bar{y}|^{2}}-\frac{1}{|x+y|^{2}}\right)\right] \omega(y) d y \\
v_{2}(x)=\int_{\mathbf{Q}}\left[\left(x_{1}-y_{1}\right)\left(\frac{1}{|x-y|^{2}}-\frac{1}{|x-\bar{y}|^{2}}\right)\right. \\
\left.+\left(x_{1}+y_{1}\right)\left(\frac{1}{|x+y|^{2}}-\frac{1}{|x+\bar{y}|^{2}}\right)\right] \omega(y) d y .
\end{aligned}
$$

Differentiating $\mathbf{P}(t)$, using the vorticity equation (2), and integrating by parts implies

$$
\mathbf{P}^{\prime}(t)=\frac{1}{m_{0}} \int_{\mathbf{Q}} x \partial_{t} \omega(t, x) d x=\frac{1}{m_{0}} \int_{\mathbf{Q}} v(t, x) \omega(t, x) d x .
$$

Furthermore, according to the modified Biot-Savart law (20), we obtain

$$
\begin{aligned}
& P_{1}^{\prime}=\frac{1}{m_{0}} \iint_{\mathbf{Q}^{2}}\left[-\left(x_{2}-y_{2}\right)\left(\frac{1}{|x-y|^{2}}-\frac{1}{|x+\bar{y}|^{2}}\right)\right. \\
& \left.+\left(x_{2}+y_{2}\right)\left(\frac{1}{|x-\bar{y}|^{2}}-\frac{1}{|x+y|^{2}}\right)\right] \omega(x) \omega(y) d x d y \\
& P_{2}^{\prime}=\frac{1}{m_{0}} \iint_{\mathbf{Q}^{2}}\left[\left(x_{1}-y_{1}\right)\left(\frac{1}{|x-y|^{2}}-\frac{1}{|x-\bar{y}|^{2}}\right)\right. \\
& \left.+\left(x_{1}+y_{1}\right)\left(\frac{1}{|x+y|^{2}}-\frac{1}{|x+\bar{y}|^{2}}\right)\right] \omega(x) \omega(y) d x d y .
\end{aligned}
$$

Interchanging the coordinates, $x \leftrightarrow y$, yields

$$
\begin{aligned}
\iint_{\mathbf{Q}^{2}}\left(x_{2}-y_{2}\right)\left(\frac{1}{|x-y|^{2}}-\frac{1}{|x+\bar{y}|^{2}}\right) \omega(x) \omega(y) d x d y \\
=-\iint\left(x_{2}-y_{2}\right)\left(\frac{1}{|x-y|^{2}}-\frac{1}{|x+\bar{y}|^{2}}\right) \omega(x) \omega(y) d x d y
\end{aligned}
$$


so

$$
\iint_{\mathbf{Q}^{2}}\left(x_{2}-y_{2}\right)\left(\frac{1}{|x-y|^{2}}-\frac{1}{|x+\bar{y}|^{2}}\right) \omega(x) \omega(y) d x d y=0 .
$$

In a similar manner, we see that

$$
\iint_{\mathbf{Q}^{2}}\left(x_{1}-y_{1}\right)\left(\frac{1}{|x-y|^{2}}-\frac{1}{|x-\bar{y}|^{2}}\right) \omega(x) \omega(y) d x d y=0 .
$$

We conclude that relation (21) can be now written as

$$
\begin{aligned}
& P_{1}^{\prime}=\frac{1}{m_{0}} \iint_{\mathbf{Q}^{2}} \frac{4 x_{1} y_{1}\left(x_{2}+y_{2}\right)}{|x-\bar{y}|^{2}|x+y|^{2}} \omega(x) \omega(y) d x d y \\
& P_{2}^{\prime}=-\frac{1}{m_{0}} \iint_{\mathbf{Q}^{2}} \frac{4 x_{2} y_{2}\left(x_{1}+y_{1}\right)}{|x+y|^{2}|x+\bar{y}|^{2}} \omega(x) \omega(y) d x d y .
\end{aligned}
$$

The first thing to remark is that $P_{1}$ is increasing and $P_{2}$ is decreasing.

The second main ingredient is conservation of energy. When the velocity lies in $L^{2}$, its norm is equivalent to the quantity

$$
E_{0}=-\frac{1}{2 \pi} \iint_{\mathbb{R}^{2} \times \mathbb{R}^{2}} \log |x-y| \omega(x) \omega(y) d x d y .
$$

However, it can be seen directly that the latter integral is a constant of the motion. Thanks to the symmetry, a few changes of coordinates reduce the integration to the first quadrant

$$
E_{0}=\frac{2}{\pi} \iint_{\mathbf{Q}^{2}} \log \frac{|x-\bar{y}||x+\bar{y}|}{|x-y||x+y|} \omega(x) \omega(y) d x d y .
$$

The kernel is nonnegative, since we can write

$$
\begin{aligned}
\log \frac{|x-\bar{y}||x+\bar{y}|}{|x-y||x+y|} & =\frac{1}{2} \log \frac{|x-\bar{y}|^{2}|x+\bar{y}|^{2}}{|x-y|^{2}|x+y|^{2}} \\
& =\frac{1}{2} \log \left(1+\frac{|x-\bar{y}|^{2}|x+\bar{y}|^{2}-|x-y|^{2}|x+y|^{2}}{|x-y|^{2}|x+y|^{2}}\right) \\
& =\frac{1}{2} \log \left(1+\frac{16 x_{1} y_{1} x_{2} y_{2}}{|x-y|^{2}|x+y|^{2}}\right) .
\end{aligned}
$$

Taking $1 / p+1 / q=1$, with $1<q<2$, Hölder's inequality along with relation (22) imply

$$
E_{0}^{p} \leq C m_{0} P_{1}^{\prime} I^{1 /(q-1)}
$$


in which

$$
I \equiv \iint_{\mathbf{Q}^{2}}\left[\frac{|x-\bar{y}|^{2}|x+y|^{2}}{x_{1} y_{1}\left(x_{2}+y_{2}\right)}\right]^{q-1}\left[\log \frac{|x-\bar{y}||x+\bar{y}|}{|x-y||x+y|}\right]^{q} \omega(x) \omega(y) d x d y .
$$

In the following, we will derive an upper bound for the integral $I$.

Since the logarithm grows more slowly than any power, given $0<\alpha<1$, there is a constant $C_{\alpha}$ such that $\log (1+z) \leq C_{\alpha} z /(1+z)^{\alpha}$, for all $z>0$. Therefore, using (23), the logarithm has the bound

$$
\begin{aligned}
\log \left(1+\frac{16 x_{1} y_{1} x_{2} y_{2}}{|x-y|^{2}|x+y|^{2}}\right) & \leq C \frac{x_{1} y_{1} x_{2} y_{2}}{|x-y|^{2}|x+y|^{2}}\left[\frac{|x-\bar{y}|^{2}|x+\bar{y}|^{2}}{|x-y|^{2}|x+y|^{2}}\right]^{-\alpha} \\
& =C \frac{x_{1} y_{1} x_{2} y_{2}}{|x-y|^{2(1-\alpha)}|x+y|^{2(1-\alpha)}|x-\bar{y}|^{2 \alpha}|x+\bar{y}|^{2 \alpha}} .
\end{aligned}
$$

From (25), this leads to the upper bound

$$
\begin{array}{r}
I \leq C \iint_{\mathbf{Q}^{2}} \frac{x_{1} y_{1}\left(x_{2} y_{2}\right)^{q}|x+y|^{2 \alpha q-2}}{\left(x_{2}+y_{2}\right)^{q-1}|x-y|^{2 q(1-\alpha)}|x+\bar{y}|^{2 \alpha q}|x-\bar{y}|^{2-2 q(1-\alpha)}} \\
\times \omega(x) \omega(y) d x d y .
\end{array}
$$

If we agree to take $\alpha=1 / q$, then this simplifies to

$$
I \leq C \iint_{\mathbf{Q}^{2}} \frac{x_{1} y_{1}\left(x_{2} y_{2}\right)^{q}}{\left(x_{2}+y_{2}\right)^{q-1}|x-y|^{2(q-1)}|x+\bar{y}|^{2}|x-\bar{y}|^{2(2-q)}} \omega(x) \omega(y) d x d y .
$$

Now the trivial inequalities

$$
x_{1} y_{1} \leq\left(x_{1}+y_{1}\right)^{2} \leq|x+\bar{y}|^{2} \quad \text { and } \quad x_{2} y_{2} \leq\left(x_{2}+y_{2}\right)^{2} \leq|x-\bar{y}|^{2}
$$

ensure that

$$
I \leq C \iint_{\mathbf{Q}^{2}} \frac{\left(x_{2}+y_{2}\right)^{3(q-1)}}{|x-y|^{2(q-1)}} \omega(x) \omega(y) d x d y .
$$

If $q \leq 6 / 5$, so that $5(q-1) \leq 1$, we can apply Hölder's inequality to get

$$
I \leq C I_{1}^{1-5(q-1)} I_{2}^{2(q-1)} I_{3}^{3(q-1)},
$$

with

$$
\begin{gathered}
I_{1}=\iint_{\mathbf{Q}^{2}} \omega(x) \omega(y) d x d y, \quad I_{2}=\iint_{\mathbf{Q}^{2}} \frac{1}{|x-y|} \omega(x) \omega(y) d x d y, \\
I_{3}=\iint_{\mathbf{Q}^{2}}\left(x_{2}+y_{2}\right) \omega(x) \omega(y) d x d y .
\end{gathered}
$$


From (19), we have that $I_{1}=m_{0}^{2}$. Lemma 2.1 tells us that $I_{2} \leq C m_{0}^{3 / 2} M_{0}^{1 / 2}$. Also, the monotonicity of $P_{2}$ gives $I_{3} \leq C m_{0}^{2} P_{2}(0)$. Altogether, we have the bound

$$
I \leq C(q) m_{0}^{2}\left[\frac{M_{0} P_{2}(0)^{3}}{m_{0}}\right]^{q-1} .
$$

Going back to (24), we obtain

$$
P_{1}^{\prime} \geq C_{0} \equiv C(q)\left[\frac{E_{0}}{m_{0}^{2}}\right]^{1 /(q-1)} \frac{E_{0}}{M_{0} P_{2}(0)^{3}},
$$

so that

$$
P_{1}(t) \geq P_{1}(0)+C_{0} t
$$

This completes the proof of Theorem 3.1.

\section{APPENDIX}

This appendix provides another proof of Proposition 2.1. In order to estimate the decay of the mass of vorticity far from the center of mass, we introduce the higher momenta:

$$
m_{n}(t)=\int|x|^{4 n} \omega(t, x) d x .
$$

Although these are not conserved quatities, a recursive estimate holds for their derivatives leading to the following result.

Lemma A.1. There exists a constant $C_{0}$ such that for any $n \geq 1$

$$
m_{n}(t) \leq m_{0}\left(d_{0}^{4}+C_{0} i_{0} n t\right)^{n} .
$$

Assume, for the moment, that Lemma A.1 is true and let us use it to prove Proposition 2.1. Fix $k \geq 1$, and suppose that

$$
r^{4} \geq 2\left[d_{0}^{4}+C_{0} i_{0} k t \log (2+t)\right] .
$$

Choose $n \geq k / 4$ in such a way that

$$
k \log (2+t)-1<n \leq k \log (2+t) .
$$

Recalling that the vorticity remains nonnegative during the motion, we have using (26), (27), and (28)

$$
\begin{aligned}
\int_{|x| \geq r} \omega(t, x) d x & \leq \frac{m_{n}(t)}{r^{4 n}} \leq \frac{m_{0}}{r^{k}} \frac{\left(d_{0}^{4}+C_{0} i_{0} n t\right)^{n}}{r^{4 n-k}} \\
& \leq \frac{m_{0}}{r^{k}} 2^{k / 4-n}\left[d_{0}^{4}+C_{0} i_{0} k t \log (2+t)\right]^{k / 4}
\end{aligned}
$$


Note that by $(28)$, we have that $2^{n+1} \geq(2+t)^{k \log 2}$. This means that the right-hand side can be bounded above by $C\left(i_{0}, d_{0}, m_{0}, k\right) / r^{k}$ when (27) holds, and so Proposition 2.1 follows.

Proof of Lemma A.1. Using the vorticity equation (2) and the Biot-Savart law (3) as in (7), we have

$$
m_{n}^{\prime}(t)=\frac{2 n}{\pi} \iint \frac{\left\langle x,(x-y)^{\perp}\right\rangle}{|x-y|^{2}}|x|^{4 n-2} \omega(t, x) \omega(t, y) d x d y .
$$

We define

$$
K(x, y)=\left\langle x,(x-y)^{\perp}\right\rangle\left(\frac{1}{|x-y|^{2}}-\frac{1}{|x|^{2}}\right) .
$$

Since the center of mass is at the origin, we can write

$$
m_{n}^{\prime}(t)=\frac{2 n}{\pi} \iint K(x, y)|x|^{4 n-2} \omega(t, x) \omega(t, y) d x d y
$$

Let us consider the following partition of the plane:

$$
\begin{aligned}
& A_{1}=\left\{(x, y):|y| \leq\left(1-\frac{1}{2 n}\right)|x|\right\}, \\
& A_{2}=\left\{(x, y):\left(1-\frac{1}{2 n}\right)|x|<|y|<\left(1-\frac{1}{2 n}\right)^{-1}|x|\right\}, \\
& A_{3}=\left\{(x, y):|x| \leq\left(1-\frac{1}{2 n}\right)|y|\right\} .
\end{aligned}
$$

Then, we have

$$
m_{n}^{\prime}(t)=\alpha_{1}(t)+\alpha_{2}(t)+\alpha_{3}(t)
$$

with

$$
\alpha_{i}=\frac{2 n}{\pi} \iint_{A_{i}} K(x, y)|x|^{4 n-2} \omega(t, x) \omega(t, y) d x d y .
$$

We will study each of these three terms.

First, assume that $(x, y) \in A_{1}$ and write

$$
K(x, y)=\left\langle y,(x-y)^{\perp}\right\rangle \frac{\langle y, 2 x-y\rangle}{|x-y|^{2}|x|^{2}} .
$$

Since $|x-y| \geq|x| / 2 n$ and $|2 x-y| \leq 3|x|$, we have the inequality

$$
|K(x, y)| \leq \frac{|y|^{2}|2 x-y|}{|x|^{2}|x-y|} \leq 6 n \frac{|y|^{2}}{|x|^{2}},
$$

and we obtain the bound

$$
\left|\alpha_{1}(t)\right| \leq \frac{12 n^{2}}{\pi} \iint_{A_{1}}|x|^{4(n-1)}|y|^{2} \omega(t, x) \omega(t, y) d x d y \leq \frac{12 n^{2}}{\pi} i_{0} m_{n-1}(t)
$$


Now, assume that $(x, y) \in A_{3}$. This implies that $|x-y| \geq|y| / 2 n$ and $(1-1 / 2 n)|y| /|x| \geq 1$. The kernel $K(x, y)$ may be written as

$$
K(x, y)=\frac{\left\langle x,(x-y)^{\perp}\right\rangle}{|x-y|^{2}}+\frac{\left\langle x, y^{\perp}\right\rangle}{|x|^{2}},
$$

and we deduce that on $A_{3}$

$$
|K(x, y)| \leq \frac{|x|}{|x-y|}+\frac{|y|}{|x|} \leq 2 n \frac{|y|^{2}}{|x|^{2}}
$$

It follows that

$$
\left|\alpha_{3}(t)\right| \leq \frac{4 n^{2}}{\pi} i_{0} m_{n-1}(t)
$$

Finally, we split the integral over $A_{2}$ into two terms

$$
\alpha_{2}(t)=I_{1}(t)+I_{2}(t)
$$

where

$$
\begin{aligned}
& I_{1}(t)=-\frac{2 n}{\pi} \iint_{A_{2}}|x|^{4 n-2} \frac{\left\langle x, y^{\perp}\right\rangle}{|x-y|^{2}} \omega(t, x) \omega(t, y) d x d y, \\
& I_{2}(t)=\frac{2 n}{\pi} \iint_{A_{2}}|x|^{4(n-1)}\left\langle x, y^{\perp}\right\rangle \omega(t, x) \omega(t, y) d x d y .
\end{aligned}
$$

In the region $A_{2}$, we have $|x| \leq 2|y|$, and we can bound the second contribution, $I_{2}(t)$, by

$$
\left|I_{2}(t)\right| \leq \frac{4 n}{\pi} i_{0} m_{n-1}(t)
$$

Now, observe that the region $A_{2}$ is symmetric with respect to the diagonal and that

$$
H(x, y) \equiv \frac{\left\langle x, y^{\perp}\right\rangle}{|x-y|^{2}}=-H(y, x) .
$$

The integral $I_{1}(t)$ can be therefore rewritten as

$$
I_{1}(t)=-\frac{n}{\pi} \iint_{A_{2}} H(x, y)\left(|x|^{4 n-2}-|y|^{4 n-2}\right) \omega(t, x) \omega(t, y) d x d y .
$$

To evaluate this integral, we first use the following identity

$$
|x|^{4 n-2}-|y|^{4 n-2}=\langle x-y, x+y\rangle \sum_{j=0}^{2 n-2}|x|^{4 n-4-2 j}|y|^{2 j} .
$$


Thus, in the region $A_{2}$, we find

$$
\begin{aligned}
\left.|| x\right|^{4 n-2}-|y|^{4 n-2} \mid & \leq 3|y||x-y||x|^{4(n-1)} \sum_{j=0}^{2 n-2}\left(1-\frac{1}{2 n}\right)^{-2 j} \\
& \leq 6 n|y||x-y||x|^{4(n-1)}
\end{aligned}
$$

On the other hand, we note that

$$
|H(x, y)|=\frac{\left|\left\langle x-y, y^{\perp}\right\rangle\right|}{|x-y|^{2}} \leq \frac{|y|}{|x-y|} .
$$

Combining the two last estimates yields

$$
\left|I_{1}(t)\right| \leq \frac{6 n^{2}}{\pi} i_{0} m_{n-1}(t)
$$

Summing up the bounds for $\alpha_{1}, \alpha_{3}, I_{1}$, and $I_{2}$, and then using Hölder's inequality we get

$$
m_{n}^{\prime}(t) \leq C_{0} i_{0} n^{2} m_{n-1}(t) \leq C_{0} i_{0} n^{2} m_{0}^{1 / n} m_{n}(t)^{1-1 / n} .
$$

It follows that $m_{n}(t)$ can be estimated as claimed in (26).

\section{ACKNOWLEDGMENTS}

T.C.S. was supported by the National Science Foundation under grant number DMS-9500284.

\section{REFERENCES}

[1] Bahouri, H. and J.-Y. Chemin. Equations de transport relatives à des champs de vecteurs non-lipschitziens et mécanique des fluides. Arch. Rat. Mech. Anal. 127 (1994), 159-182.

[2] Batchelor, G.K. An introduction to fluid dynamics. Cambridge University Press, Cambridge (1967).

[3] Bertozzi, A. and P. Constantin. Global regularity for vortex patches. Comm. Math. Phys. 152 (1993), 19-28.

[4] Buttke, T. A fast adaptive vortex method for patches of constant vorticity in two dimensions. J. Comp. Phys. 89 (1990), 161-186.

[5] Chemin, J.-Y. Sur le mouvement des particules d'un fluide parfait, incompressible, bidimensionnel. Inven. Math. 103 (1991), 599-629.

[6] Chemin, J.-Y. Fluides parfaits incompressibles. Astérisque. 230 (1995). 
[7] Danchin, R. Evolution temporelle d'une poche de tourbillon singulière. Comm. PDE 22 (1997), 685-721.

[8] Delort, J.-M. Existence de nappes de tourbillon en dimension deux. J. Amer. Math. Soc. 4 (1991), 553-586.

[9] DiPerna, R. and A. Majda. Concentrations in regularizations for 2-D incompressible flow. Comm. Pure Appl. Math. 40 (1987), 301-345.

[10] Lamb, H. Hydrodynamics, 6th ed. Cambridge University Press, Cambridge (1932).

[11] Lopes Filho, M. and H. Nussenzveig Lopes. An extension of C. Marchioro's bound on the growth of a vortex patch to flows with $L^{p}$ vorticity. SIAM J. Math. Anal. 293 (1998) 596-599.

[12] Marchioro, C. Bounds on the growth of the support of a vortex patch. Comm. Math. Phys. 164 (1994), 507-524.

[13] Serfati, P. Borne en temps des caractéristiques de l'équation d'Euler 2D à tourbillon positif et localisation pour le modèle point-vortex. Preprint.

[14] Wolibner, W. Un théorème sur l'existence du mouvement plan d'un fluide parfait, homogène, incompressible, pendant un temps infiniment long. Math. Z. 37 (1933), 698-726.

[15] Yudovitch, V.I. Non-stationary flow of an ideal incompressible liquid. Zh. Vych. Mat. 36 (1963), 1032-1066. 